\title{
Research on a Noise Reduction Method Based on DTCWT and the Cyclic Singular Energy Difference Spectrum
}

\author{
Xihui Chen 1,2,* - Gang Cheng2,4 - Ning Liu ${ }^{3}$ - Xinhui Shi 1 - Wei Lou ${ }^{1}$ \\ ${ }^{1}$ Hohai University, College of Mechanical and Electrical Engineering, China \\ 2 China University of Mining and Technology, School of Mechatronic Engineering, China \\ ${ }^{3}$ China Coal Technology and Engineering Grope Shanghai Co., Ltd., China \\ 4 Shandong Zhongheng Optoelectronic Technology Co., Ltd., China
}

The gear is the most important part of the transmission system of mechanical equipment, and the monitoring and diagnosis of it can improve the reliability of mechanical equipment. However, mechanical equipment generally works in harsh working conditions. The gear vibration signal is subjected to strong noise interference in working conditions, which brings great challenges for the effective diagnosis of gear faults. This paper proposed a noise reduction method based on the dual-tree complex wavelet transform (DTCWT) and cyclic singular energy difference spectrum. First, the gear vibration signal containing strong noise interference is decomposed into a series of signal components with different frequency characteristics by using the time-frequency analysis ability of DTCWT. Then, cyclic singular energy difference spectrum is proposed based on the idea of a cascaded cycle and the successive elimination of noise interference to process each signal component with different frequency characteristics, and the termination conditions of cyclic singular energy difference spectrum can be set according to the noise interference distribution characteristics in different frequency bands. The final noise reduction of the original gear vibration signal can be realized based on signal reconstruction after the noise reduction processing of each signal components with different frequency bands. Finally, experiments are carried out to verify the effectiveness of the proposed method, which is effective and suitable for the noise reduction of the vibration signal.

Keywords: noise reduction, DTCWT, singular value decomposition, cyclic singular energy difference spectrum, vibration signal

\section{Highlights}

- A noise reduction method based on dual-tree complex wavelet transform and cyclic singular energy difference spectrum is proposed.

- The cyclic singular energy difference spectrum is proposed based on the idea of a cascaded cycle and the successive elimination of noise interference.

- $\quad$ The adaptive selection methods of some parameters are applied to the proposed cyclic singular energy difference spectrum.

- The simulated signals and experimental signals are used to verify the proposed method, which proves that the proposed method can effectively eliminate noise interference.

\section{INTRODUCTION}

The gear is the most important part of mechanical equipment and is mainly responsible for the transmission of motion and power. On-line monitoring and fault diagnosis of gear is crucial in ensuring the safe operation of mechanical equipment [1] and [2]. At present, the vibration signal analysis is the mainstream of gear fault diagnosis. However, the actual operation condition of mechanical equipment is generally harsh, the result of which is that the collected vibration signal contains a large amount of noise interference [3] and [4]. The vibration signals obtained under harsh working conditions generally contain strong noise interference, which conceals the weak fault feature produced by the gear fault and greatly affects the accuracy of the vibration signal. The difficulty of obtaining useful information from the collected vibration signals is increased under such operation conditions [5]. Therefore, the pre-processing of noise reduction is essential for the vibration signal in real working conditions.

The commonly used traditional noise reduction method includes low-pass, high-pass, and bandpass filters, but for the noise interference generated in real working conditions, no matter which filter is used, the integrity of the useful signal components will be affected [5]. With the development of signalprocessing technology, more noise reduction methods have been proposed. Wavelet transform and singular value decomposition (SVD) are more commonly used in modern signal processing. Wavelet transform can decompose the original vibration signal into several signal components with different frequency bands and different resolutions [6]; the noise reduction is then processed for each signal components with different frequency bands. Generally, noise reduction methods based on wavelet transform include wavelet modulus maximum denoising, wavelet threshold denoising, wavelet correlation denoising and similar. However, 
wavelet threshold denoising needs to determine the threshold value, and an accurate threshold setting has a great influence on noise reduction effect. Wavelet correlation denoising uses the correlation of wavelet coefficients at corresponding points on different scales to distinguish the useful signal coefficients and noise coefficients to achieve the purpose of noise reduction. However, wavelet correlation denoising has the disadvantage of a complex algorithm, and its noise reduction effect is generally limited by the judging of correlation of wavelet coefficients. The noise reduction based on SVD can eliminate noise interference according to the different contribution of useful signal and noise interference to singular values, and that has been applied in the noise reduction of vibration signal [7].

However, the noise reduction methods based on SVD are faced with the problems of phase space matrix construction and denoising order selection. The noise interference generated in real working condition is more complex, which has the characteristics of irregularity, wide frequency distribution, and similar. In order to eliminate the strong noise interference in real work conditions, combining the advantages of the noise reduction ideas based on wavelet and SVD provides a new idea for the pre-processing of noise reduction of the gear vibration signal. The strong noise interference can be decomposed into a series of signal components with different frequency bands, and the corresponding denoising criteria based on SVD can be built to eliminate the noise interference in each frequency band according to its distribution characteristics.

Nevertheless, the general wavelet transform has some shortcomings, such as frequency leakage, frequency aliasing, translation sensitivity and less direction selection, and there are also some problems such as phase space matrix construction and denoising order selection in SVD [8]. With the development of the general wavelet transform, DTCWT has been proposed. Compared with the general wavelet transform, DTCWT has the characteristics of translation invariance, small frequency aliasing and multi-direction selection [9] and [10]. Meanwhile, the selections of embedding dimension, delay parameters and denoising order involved in the noise reduction based on SVD also need to be further studied [11].

In this paper, a noise reduction method based on DTCWT and cyclic singular energy difference spectrum is proposed. The gear vibration signal with strong noise interference is decomposed into a series of signal components by DTCWT, and the strong noise interference in each frequency band can be eliminated by cyclic singular energy difference spectrum. The noise reduction of the gear vibration signal can be realized by reconstructing the signal components of each frequency band after noise reduction. Finally, the validity of the proposed method is verified by experimental signal analysis.

However, it should be noted that most equipment is not equipped with the suitable vibration sensors for state monitoring and matching acquisition systems in existing large equipment, such as shearers, heading machines, shield machines, and so on. Therefore, it is impossible to realize the long-time continuous acquisition of vibration signal in the working process of equipment. Only the external vibration sensors and acquisition system can be used for short-term experiments. However, the gear fault in the real working condition usually occurs suddenly and has a certain probability, so it is very difficult to obtain the vibration signal of gear fault in the short-term acquisition process. Therefore, this paper uses the mechanical fault simulation bench to simulate the vibration signal of the gear fault. Meanwhile, the strong noise interference in real working condition is collected. Then, the strong noise interference after energy conversion is added into the vibration signal of the gear fault, which provides a way to obtain the gear fault vibration signal under strong noise interference in real working conditions.

\section{MODEL BUILDING}

\subsection{Dual-Tree Complex Wavelet Transform}

The basis function of DTCWT consists of two distinct real wavelets, and they constitute the Hilbert transform pair. The basis function of DTCWT is shown as [12] and [13]:

$$
\varphi(t)=\varphi_{h}(t)+i \varphi_{g}(t)
$$

where $\boldsymbol{\varphi}_{h}(t)$ and $\boldsymbol{\varphi}_{g}(t)$ are two real wavelets, and $i$ is complex unit. $\varphi_{h}(t)$ is the wavelet of real tree, and $\boldsymbol{\varphi}_{g}(t)$ is the wavelet of complex tree, and $\boldsymbol{\varphi}_{h}(t)$ and $\boldsymbol{\varphi}_{g}(t)$ are mutually Hilbert transform pairs.

The wavelet transform results in a real tree for signal $\mathbf{y}(t)$ are as follows:

$$
\left\{\begin{array}{l}
\mathbf{D I}_{j}^{\mathrm{Re}}(n)=2^{j / 2} \int_{-\infty}^{+\infty} \mathbf{y}(t) \boldsymbol{\varphi}_{g}\left(2^{j} t-n\right) \mathrm{d} t \quad j=1,2, \ldots, J \\
\mathbf{C I}_{J}^{\mathrm{Re}}(n)=2^{J / 2} \int_{-\infty}^{+\infty} \mathbf{y}(t) \boldsymbol{\varphi}_{g}\left(2^{J} t-n\right) \mathrm{d} t
\end{array},\right.
$$

where $\mathbf{D I}{ }_{j}^{\mathrm{Re}}(n)$ is the high-frequency coefficients in a real tree, $\mathbf{C I}_{J}^{\mathrm{Re}}(n)$ is the low-frequency coefficients in a real tree, and $n$ is the number of data points. $j$ is the 
decomposition scale coefficient, and $J$ is the largest scale.

The wavelet transform results in a complex tree for signal $\mathbf{y}(t)$ are as follows:

$$
\left\{\begin{array}{l}
\mathbf{D I}_{j}^{\mathrm{Im}}(n)=2^{j / 2} \int_{-\infty}^{+\infty} \mathbf{y}(t) \boldsymbol{\varphi}_{h}\left(2^{j} t-n\right) \mathrm{d} t \quad j=1,2, \ldots, J \\
\mathbf{C I}_{J}^{\mathrm{Im}}(n)=2^{J / 2} \int_{-\infty}^{+\infty} \mathbf{y}(t) \boldsymbol{\varphi}_{h}\left(2^{J} t-n\right) \mathrm{d} t
\end{array},\right.
$$

where $\mathbf{D I}_{j}^{\mathrm{Im}}(n)$ is the high-frequency coefficients in a complex tree, $\mathbf{C I}_{J}^{\mathrm{Im}}(n)$ is the low-frequency coefficients in a complex tree.

The high-frequency coefficients and lowfrequency coefficients obtained with DTCWT can be further expressed as follows:

$$
\left\{\begin{array}{l}
\mathbf{D}_{j}=\mathbf{D} \mathbf{I}_{j}^{\mathrm{Re}}(n)+i \mathbf{D} \mathbf{I}_{j}^{\mathrm{Im}}(n) \quad j=1,2, \ldots, J \\
\mathbf{C}_{J}=\mathbf{C} \mathbf{I}_{J}^{\mathrm{Re}}(n)+i \mathbf{C I} \mathbf{I}_{J}^{\mathrm{Re}}(n)
\end{array},\right.
$$

where $\mathbf{D}_{j}$ is the high-frequency coefficients of DTCWT, and $\mathbf{C}_{J}$ is the low-frequency coefficients of DTCWT.

In the specific calculation process, the decomposition and reconstruction of DTCWT are implemented with a Mallet fast algorithm [14]. According to the Mallet fast decomposition algorithm, the transformation relationship of the decomposition coefficients between scale $j$ and scale $j+1$ is as follows:

$$
\left\{\begin{array}{l}
\mathbf{C I}_{j+1}^{\mathrm{Re}}(k)=\sum_{m} \mathbf{h}_{0}(m-2 k) \mathbf{C} \mathbf{J}_{j}^{\mathrm{Re}}(m) \\
\mathbf{D I}_{j+1}^{\mathrm{Re}}(k)=\sum_{m} \mathbf{h}_{1}(m-2 k) \mathbf{D} \mathbf{I}_{j}^{\mathrm{Re}}(m) \\
\mathbf{C I}_{j+1}^{\mathrm{Im}}(k)=\sum_{m} \mathbf{g}_{0}(p-2 k) \mathbf{C} \mathbf{J}_{j}^{\mathrm{Im}}(p) \\
\mathbf{D I}_{j+1}^{\mathrm{Im}}(k)=\sum_{m} \mathbf{g}_{1}(p-2 k) \mathbf{D I} \mathbf{I}_{j}^{\mathrm{Im}}(p) \\
\mathbf{C I}_{j}^{\mathrm{Re}}(k)=\sum_{m} \overline{\mathbf{h}}_{0}(k-2 m) \mathbf{C \mathbf { I } _ { j + 1 } ^ { \mathrm { Re } }}(m)+\sum_{m} \overline{\mathbf{h}}_{1}(k-2 m) \mathbf{D I}_{j+1}^{\mathrm{Re}}(m) \\
\mathbf{C I}_{j}^{\mathrm{Im}}(k)=\sum_{m} \overline{\mathbf{g}}_{0}(k-2 p) \mathbf{C} \mathbf{I}_{j+1}^{\mathrm{Im}}(p)+\sum_{m} \overline{\mathbf{g}}_{1}(k-2 p) \mathbf{D I} \mathbf{I}_{j+1}^{\mathrm{Im}}(p)
\end{array},\right.
$$

where $\mathbf{h}_{0}$ and $\mathbf{h}_{1}$ are the low-pass filter and high-pass filter of real tree wavelet, and $\mathbf{g}_{0}$ and $\mathbf{g}_{1}$ are the lowpass filter and high-pass filter of complex tree wavelet. $m$ is the length of the filter of real tree wavelet, and $p$ is the length of the filter of complex tree wavelet. $k$ is the number of data points. $\overline{\mathbf{h}}_{0}, \overline{\mathbf{h}}_{1}, \overline{\mathbf{g}}_{0}$ and $\overline{\mathbf{g}}_{1}$ are the corresponding reconstruction filters, respectively. The decomposition and reconstruction process of DTCWT can be represented as Fig. 1 [15].

The original vibration signal containing strong noise interference is decomposed into a series of signal components with different frequency characteristics. Next, a cyclic singular energy difference spectrum is used to realize the pre-processing of noise interference in each frequency band.

\subsection{Cyclic Singular Energy Difference Spectrum}

\subsubsection{Noise Reduction Principle Based on SVD}

The noise reduction idea based on SVD is according to the singular value difference contributed by useful signal and noise interference [16]. Assuming that the vibration signal includes noise interference is $\mathbf{x}=\left(x_{1}, x_{2}, \ldots, x_{N}\right)$, where $N$ is the number of data points. The phase space matrix of the vibration signal $\mathbf{x}$ can be constructed as follows:

$$
\mathbf{A}=\left[\begin{array}{cccc}
x_{1} & x_{2} & \ldots & x_{m} \\
x_{1+\tau} & x_{2+\tau} & \ldots & x_{m+\tau} \\
\vdots & \vdots & & \vdots \\
x_{1+(d-1) \tau} & x_{2+(d-1) \tau} & \ldots & x_{m+(d-1) \tau}
\end{array}\right]_{m \times d},
$$

where $m=N-(d-1) \tau$, and $\tau$ is the delay parameter, $d$ is the number of matrix rows, that is embedding dimension.

The construction of phase space matrix is the foundation of the noise reduction based on SVD, and the one-dimensional time-domain vibration signal can be transformed into a phase space matrix with multiple-dimensions. In the phase space matrix, the data in the $(n+1)^{\text {th }}$ row always lags behind the $\tau$ data points compared with the data in the $n^{\text {th }}$ row, and $\tau$ is the delay parameter. For example, in Eq. (6), the data in the first row of phase space matrix $\mathbf{A}$ is $\left(x_{1}, x_{1}, \ldots, x_{m}\right)$, and the data in the second row of phase space matrix $\mathbf{A}$ is $\left(x_{1+\tau}, x_{2+\tau}, \ldots, x_{m+\tau}\right)$. If the vibration signal is composed of the ideal signal without noise interference, the data between the adjacent two rows of phase space matrix will be highly correlated. That is an ill-conditioned matrix, and the singular values with large values for this matrix are thought to be contributed by a useful signal. If the signal is composed of noise interference, the data between the adjacent two rows have a small correlation, which is a well-conditioned full-rank matrix, and the singular value structure with uniform numerical distribution can be produced. Therefore, it can be considered that the larger singular value is contributed by both useful signal and noise interference, and the smaller singular value is contributed entirely by noise interference. The noise interference can be effectively eliminated by selecting and preserving singular values. For phase matrix $\mathbf{A}^{m \times d}$, according to the theory of SVD, there must be a pair of orthogonal matrices $\mathbf{U} \in \mathbf{R}^{m \times d}$ and 


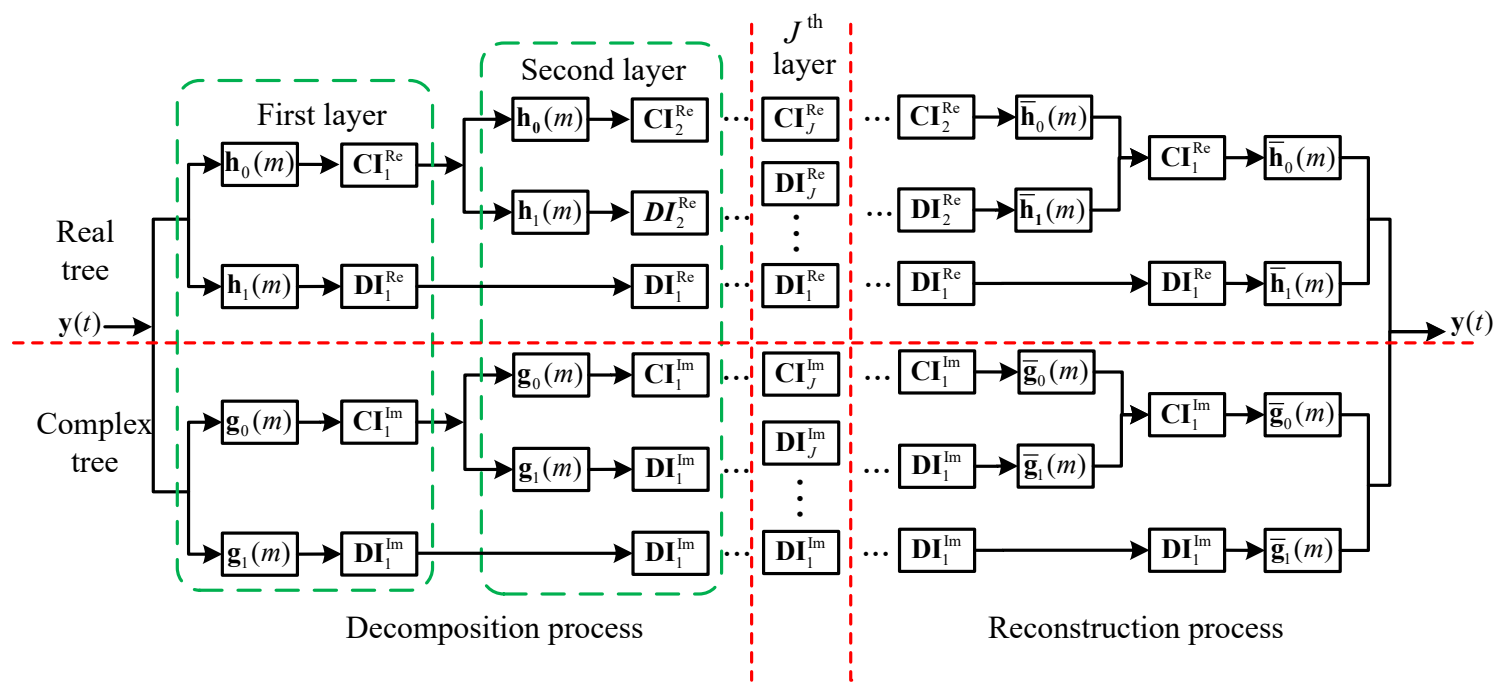

Fig. 1. The decomposition and reconstruction processes of DTCWT

$\mathbf{V} \in \mathbf{R}^{d \times d}$, which satisfy $\mathbf{U}^{T} \mathbf{U}=\mathbf{I}_{d}$ and $\mathbf{V}^{T} \mathbf{V}=\mathbf{V} \mathbf{V}^{T}=\mathbf{I}_{d}$ [17]. The phase matrix can be expressed as follows:

$$
\mathbf{A}=\mathbf{U S V}^{T} \text {, }
$$

where $\mathrm{S}=\left\{\lambda_{1}, \lambda_{2}, \ldots, \lambda_{h}\right\}, \lambda_{1} \geq \lambda_{2} \geq \ldots \geq \lambda_{h} \geq 0$ is a diagonal matrix with $(1+(d-1) \tau) \times m$ dimension, and they are the singular values of phase space matrix $\mathbf{A}$. $\mathbf{U}$ is the left singular matrix of $\mathbf{A}$, and $\mathbf{V}$ is the right singular matrix of $\mathbf{A}$.

Next, the singular values contributed by the useful signal are retained, and that contributed by noise interference are set to zero. The singular value diagonal matrix is converted to $\tilde{\mathbf{S}}=\left(\lambda_{1}, \lambda_{2}, \ldots, \lambda_{k}, 0, \ldots, 0\right) \quad k \leq h=(1+(d-1) \tau) \times m$. The inverse process of SVD is performed according to Eq. (8) on the basis of the left singular matrix $\mathbf{U} \in \mathbf{R}^{m \times d}$ and right singular matrix $\mathbf{V} \in \mathbf{R}^{d \times d}$, and the phase space eliminated noise interference can be obtained. Further, the inverse process of constructing the phase space matrix is conducted, and the noise reduction can be realized.

$$
\tilde{\mathbf{A}}=\mathbf{U} \tilde{\mathbf{S}} \mathbf{V}^{T} \text {. }
$$

Through the analysis of the noise reduction theory of SVD, selecting the denoising order, that is, determining the number of the retained singular values is the key factor that affects the effect of noise reduction.

\subsubsection{Cyclic Singular Energy Difference Spectrum}

The key to eliminating noise interference is the selection of denoising order. However, at present, most noise reduction methods based on SVD are used to eliminate the noise interference one-time by selecting denoising order. However, the large singular value may be contributed by useful signal and noise interference, and the smaller mutation point of the maximum singular value may also contain the useful signal. So, the idea of one-time completion of noise reduction may lead to the noise interference being unable to be eliminated completely or useful signals being mistakenly eliminated. Therefore, the noise reduction method called cyclic singular energy difference spectrum is proposed based on the idea of a cascaded cycle and successive elimination of noise interference. In each cycle of the noise reduction process, only the definite noise interference is eliminated each time, and though the cycle process, the strong noise interference can be eliminated successively step by step. In the noise reduction process of using cyclic singular energy difference spectrum, each cyclic noise reduction process needs to select the delay parameter, embedding dimension and denoising order. The selection of relevant parameters of the proposed method is as follows:

\section{(1) Selection of delay parameter}

In this paper, the mutual information function method is used to determine the delay parameter, and the formula of mutual information function is as follows [18]:

$$
\mathbf{I}(\tau)=\sum_{i=1}^{N-\tau} \mathbf{p}\left(x_{i}, x_{i+\tau}\right) \ln \frac{\mathbf{p}\left(x_{i}, x_{i+\tau}\right)}{\mathbf{p}\left(x_{i}\right) p\left(x_{i+\tau}\right)},
$$

where $\mathbf{p}\left(x_{i}\right)$ is the probability of numerical occurrence of the $i^{\text {th }}$ data point in a vibration signal, and $\mathbf{p}\left(x_{i+\tau}\right)$ 
is the probability of numerical occurrence of the $(i+\tau)^{\text {th }}$ data point. $\mathbf{p}\left(x_{i}, x_{i+\tau}\right)$ is the joint probability of $\mathbf{p}\left(x_{i}\right)$ and $\mathbf{p}\left(x_{i+\tau}\right)$, and it represents the probability of the numerical occurrence of $x_{i+\tau}$ after the numerical occurrence of $x_{i}$. The selection of the delay parameter is defined according to the corresponding value of the first minimum to Eq. (9) [19].

(2) Selection of embedding dimension

In this paper, the embedding dimension of the phase space is selected by the follows [20]:

$$
\mathbf{E}(d)=\frac{1}{N-d \tau} \sum_{i=1}^{N-d \tau} \frac{\left\|\mathbf{X}_{i}(d+1)-\mathbf{X}_{n(i, d)}(d+1)\right\|}{\left\|\mathbf{X}_{i}(d)-\mathbf{X}_{n(i, d)}(d)\right\|},
$$

where $\mathbf{X}_{i}(d)$ is the $i^{\text {th }}$ reconstruction vector of the constructed phase space when the embedding dimension is $d$ and delay parameter $\tau$ is determined, and $\mathbf{X}_{n(i, d)}(d)$ is the nearest neighbour of $\mathbf{X}_{i}(d)$. The nearest neighbour $\mathbf{X}_{n(i, d)}(d)$ is the reconstruction vector with the minimum Euclidean distance between the other reconstruction vectors of the constructed phase space and $\mathbf{X}_{i}(d)$. When the delay parameter $\tau$ is determined, the value of $\mathbf{E}(d)$ is only related to the embedding dimension $d$. To define $\mathbf{E}_{1}(d)=\mathbf{E}(d) / \mathbf{E}(d+1)$, it is generally believed that the value of $d$ is the appropriate embedding dimension when $\mathbf{E}_{1}(d)$ changes slowly as the increasing of $d$ [16].

\section{(3) Selection of denoising order}

In this paper, a new selection method of denoising order is proposed: singular energy difference spectrum. The singular energy difference spectrum is to calculate the energy difference between each singular value and its adjacent singular values. According to the characteristic that the singular values of noise interference and useful signal are quite different, the signal component mutation (useful signal and noise interference) occurs at the abrupt change of singular value, which is the inevitable result of the difference in correlation between useful signal and noise interference. Meanwhile, calculating the energy of singular values can amplify the difference between adjacent singular values, and the mutation between adjacent singular values can be increased. It is easier to detect the mutation point of singular values, and the determined mutation point can better represent the mutation of signal components at here. Furthermore, all extreme points of the singular energy difference spectrum and their corresponding sequence numbers can be obtained, and the average value of all extreme points can be calculated. The maximum singular value sequence number of the extreme point with greater than the average value can be taken as the denoising order, and the singular values smaller than the denoising order can be determined to be completely contributed by noise interference. The detailed calculation process is as follows:

SVD is performed on the phase space matrix [21], and the obtained singular value sequence is $\mathbf{S}=\left\{\lambda_{1}, \lambda_{2}, \ldots, \lambda_{h}\right\}$, and $\lambda_{1} \geq \lambda_{2} \geq \ldots \geq \lambda_{h} \geq 0$, and the energy of singular value can be expressed as follows:

$$
\mathbf{E}_{i}=\lambda_{i}^{2},
$$

where $\mathbf{E}_{i}$ is the energy of the $i^{\text {th }}$ singular value.

The difference spectrum of singular energy can be expressed as follows:

$$
\mathbf{b}_{i}=\lambda_{i}^{2}-\lambda_{i+1}^{2}, i=1,2, \ldots, h .
$$

The average of the difference spectrum of the singular energy is calculated.

$$
\overline{\mathbf{p}}=\left(\lambda_{i}^{2}-\lambda_{i+1}^{2}\right) / \sum_{i=1}^{h} \lambda_{i}^{2} .
$$

The extreme value point $b_{i}$ of singular energy difference spectrum represents the mutation of the signal components at the $i^{\text {th }}$ singular value. The singular value sequence numbers of all extreme points that are is greater than the average value are found. The maximum singular value sequence number of the extreme point with greater than the average value $\overline{\mathbf{p}}$ is taken as the denoising order of one-time cycle. It can be found that the denoising order is a mutation point of singular value, which represents the signal component mutation. It is not the biggest mutation point, and it is obtained according to the average value. The singular values smaller than the denoising order can be determined to be essentially contributed by noise interference.

\section{(4) Algorithm process}

The proposed cyclic singular energy difference spectrum is introduced on the basis of selection methods of delay parameter, embedding dimension and denoising order.

Step 1: According to the characteristics of vibration signal under strong noise interference, the delay parameter and embedding dimension are selected by Eqs. (9) and (10), and the phase space matrix can be constructed;

Step 2: The singular energy difference spectrum can be obtained according to Eqs. (11) and (12), and the average value of those can be further obtained;

Step 3: The singular value sequence numbers whose abrupt extreme values are greater than their average value in singular energy difference spectrum 
are obtained, and the maximum sequence number is defined as the denoising order, and the singular values whose sequence number are greater than denoising order are replaced by zero;

Step 4: The inverse process of SVD is carried out, and the noise reduction signal can be reconstructed, and one-time noise reduction is completed, and there is still a part of noise interference that has not been eliminated.

Step 5: The signal after one-time noise reduction is regarded as a new signal, and it is treated as a new processed object to replace the original vibration signal. Then, the process of Steps 1 to 4 is cycled until the termination condition of noise reduction of cyclic singular energy difference spectrum is satisfied.

Step 6: In the proposed cyclic singular energy difference spectrum, the termination condition is that the maximum sequence number of the singular values whose abrupt extreme values detected in Step 3 are greater than the average value of those is less than a certain value. When the termination condition is satisfied, the noise reduction process will be terminated after this time noise reduction. The flowchart of the proposed cyclic singular energy difference spectrum is shown in Fig. 2.

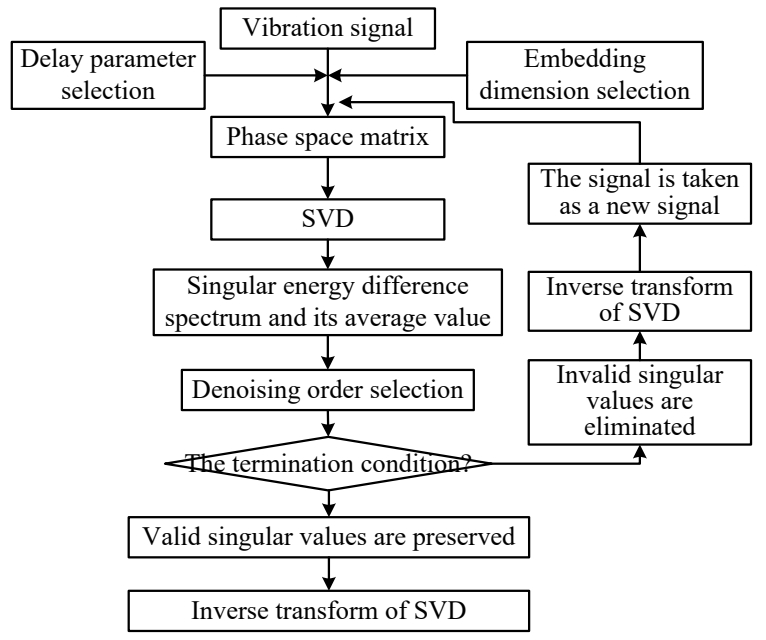

Fig. 2. The flowchart of the proposed cyclic singular energy difference spectrum

\section{EXPERIMENTAL ANALYSIS}

The overall analysis flowchart of the proposed method is shown in Fig. 3, and MATLAB software is used for the following analysis and processing of signals.

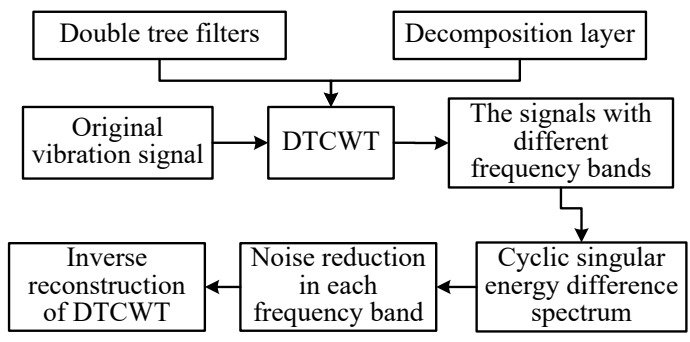

Fig. 3. The flowchart of the proposed noise reduction method

\subsection{The Analysis of the Simulated Signal}

Based on the characteristics of the vibration signal generated by gears, the simulated signal is shown as Eq. (14), and its time-domain signal and frequency spectrum are shown in Fig. 4.
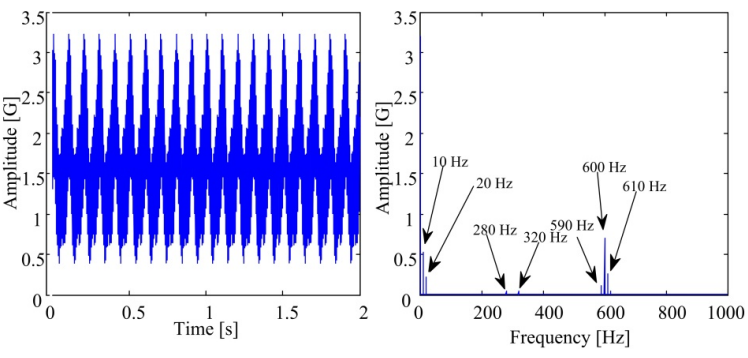

Fig. 4. The simulated signal a) time-domain signal, and b) its frequency spectrum

It can be seen from Fig. 4 that the simulated time-domain signal is more regular and have more obvious periodic components. The main frequency components are outstanding. The Gaussian white noise is used to simulate strong noise interference, and which are added into the simulated signal. The simulated signal added noise interference and its frequency spectrum are shown in Fig. 5.

$$
\left\{\begin{array}{rl}
\mathbf{y}_{0}(t)= & (1+0.8+0.4 \cos (20 \pi t)) \sin (0.1 \sin (20 \pi t)) \\
\mathbf{y}_{1}(t)= & (1+0.6+0.5 \cos (20 \pi t)+0.1 \cos (40 \pi t)) \\
& \cdot \cos (600 \pi t+0.1 \sin (20 \pi t)) \\
\mathbf{y}_{2}(t)= & 0.5(1+0.5+0.3 \cos (20 \pi t)) \\
& \cdot \sin (1200 \pi t+0.5 \sin (20 \pi t)+0.2 \sin (40 \pi t)) \\
\mathbf{y}=\mathbf{y}_{0} & +\mathbf{y}_{1}+\mathbf{y}_{2} \\
t=0: 0.0005: 2
\end{array} .\right.
$$

It can be seen from Fig. 5 that the simulated signal is completely submerged by the added noise interference. Except for the very prominent frequency points of $10 \mathrm{~Hz}$ and $600 \mathrm{~Hz}$, the other frequency components are disturbed by the noise interference. The proposed noise reduction method is used to deal 
with the simulated signal added noise interference. Firstly, DTCWT is used, and the number of the decomposition layers is important for the subsequent noise reduction effect. If the number of decomposition layers is large (more than 8), the frequency band of some decomposed signal components will be too narrow, and it is easy to cause signal loss and signal distortion. If the number of decomposition layers is small (less than 4), it will cause the similar wavelet coefficients of useful signal and noise interference can not be separated, which cause the noise reduction effect of the subsequent process to be reduced. Therefore, through multiple experiments of the decomposition effect of DTCWT, the number of decomposition layer is determined to 6 . The decomposition result of DTCWT is shown in Fig. 6.
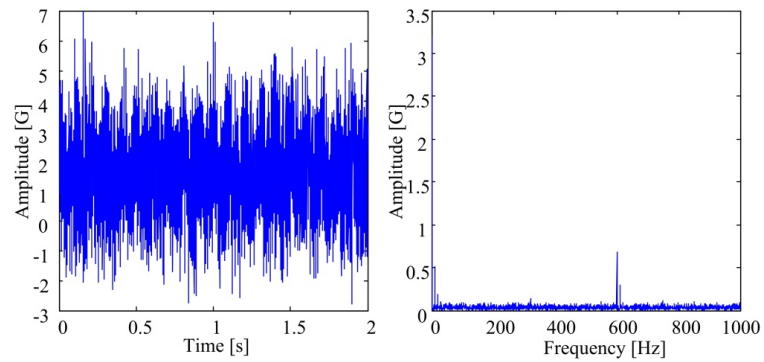

Fig. 5. The simulated signal added noise interference and its frequency spectrum
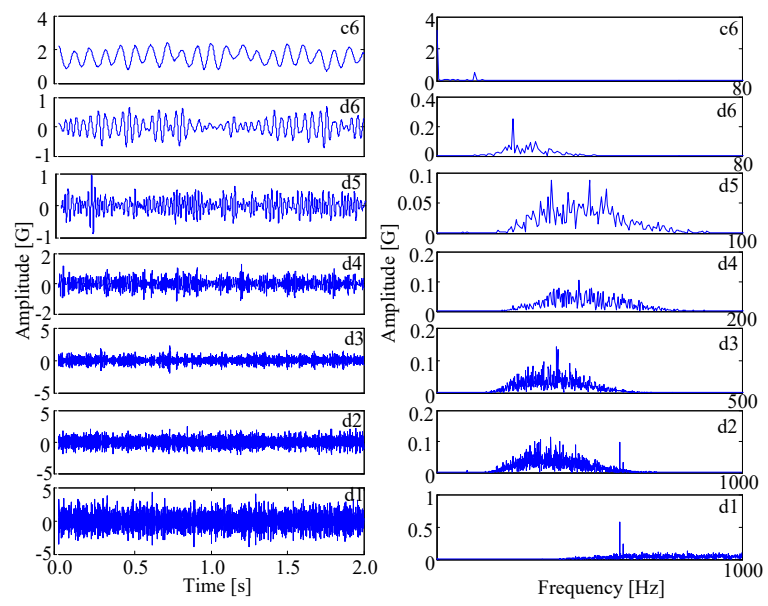

Fig. 6. The decomposition result of DTCWT

The simulated signal added noise interference is decomposed into seven signal components by DTCWT, and the corresponding noise interference is also decomposed into each signal component. For each signal component with different frequency bands, the cyclic singular energy difference spectrum is carried out. Meanwhile, the termination condition is that the denoising order determined by the last cyclic noise reduction is less than a certain value. In this way, the corresponding termination conditions can be set according to the noise distribution of each frequency band. Due to the space limitation, the noise reduction process in frequency band $\mathrm{d} 1$ is illustrated as an example, and the termination condition of this signal component is set as the denoising order being determined by the last cyclic process of noise reduction being less than 10 . The noise reduction result of the signal component of frequency band $\mathrm{d} 1$ is shown in Fig. 7.

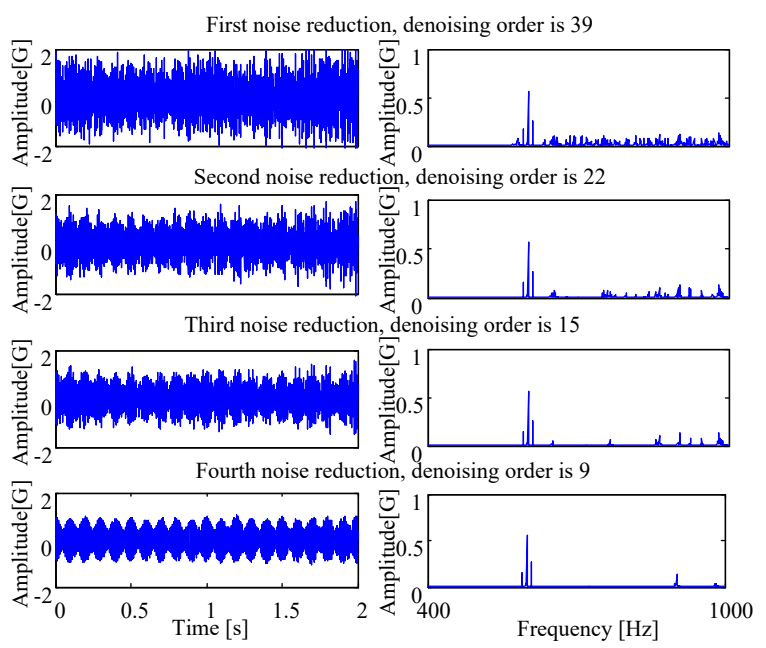

Fig. 7. Cyclic noise reduction result of the signal component of frequency band $\mathrm{d} 1$

As can be found in Fig. 7, for the signal component of frequency band $\mathrm{d} 1$, a total of four-cycle processes of noise reduction are used. With each cycle process of noise reduction, the noise interference is filtered out step by step, and the effective signal after noise reduction can be obtained. It can be found from the reduction result that the main frequency components in the frequency spectrum are $590 \mathrm{~Hz}$, $600 \mathrm{~Hz}, 900 \mathrm{~Hz}$, and $982 \mathrm{~Hz}$, which are merely the frequency components of the simulated signal (Fig. 4). It is illustrated that the proposed method can eliminate noise interference better.

Furthermore, $900 \mathrm{~Hz}$ and $982 \mathrm{~Hz}$ are regarded as the useful signal and not filtered out in the final noise reduction. However, comparatively speaking, the proposed method can eliminate the most of noise interference, it has a good noise reduction effect. For other signal components obtained by DTCWT, noise reduction is carried out according to the cyclic singular energy difference spectrum, and the final noise reduction results of the simulated signal can be obtained, and they are shown in Fig. 8. 

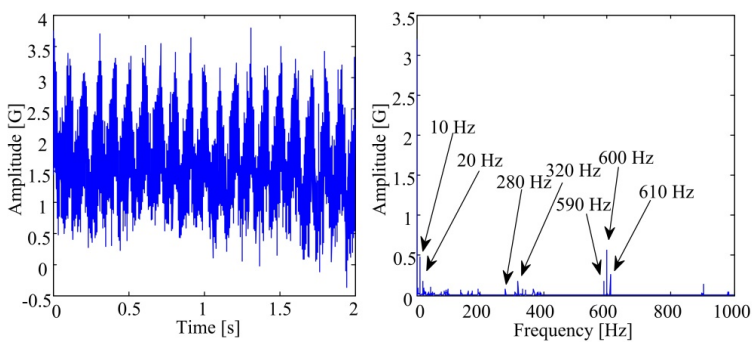

Fig. 8. Final noise reduction result of the simulated signal

It can be seen from Fig. 8 that the proposed method can effectively eliminate the strong noise interference comparing with Fig. 5. In the final noise reduction result, the main feature frequency components of the simulated signal are $10 \mathrm{~Hz}, 20$ $\mathrm{Hz}, 280 \mathrm{~Hz}, 320 \mathrm{~Hz}, 590 \mathrm{~Hz}, 600 \mathrm{~Hz}$, and $610 \mathrm{~Hz}$, and they are completely retained, indicating that the above method can better eliminate noise interference and retain the useful signal. Comparing Figs. 4 and 8, it can be found that some frequency components are not filtered out; however, the amplitude of those frequency components are relatively low, and their interference with the useful signal is limited. Next, the validity of the proposed method is verified by the processing of the gear vibration signal under strong noise interference in real working condition.

\subsection{Analysis of the Experiment Signal}

The acquisition experiment of the strong noise interference in real working conditions is carried out in the gear system of rocker arm of electric haulage shearer. The vibration signals generated by the gear system are collected when it is running under no-load and normal cutting and the strong noise interference in real working condition can be extracted, and the acquisition experiment is shown in Fig. 9, and the strong noise interference in real working conditions is shown in Fig. 10.

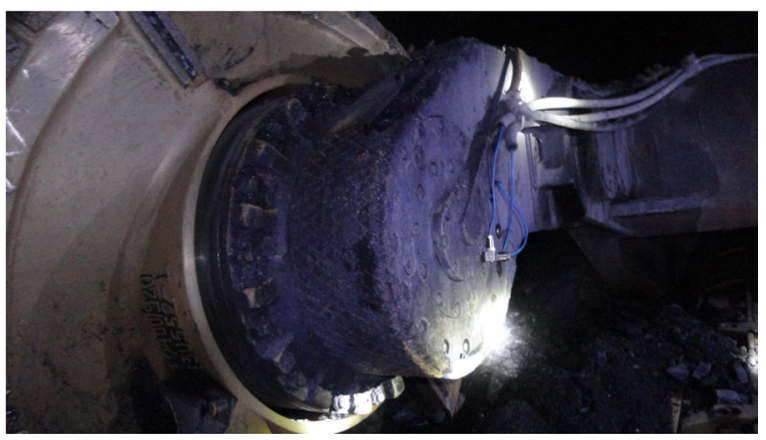

Fig. 9. The acquisition experiment of strong noise interference

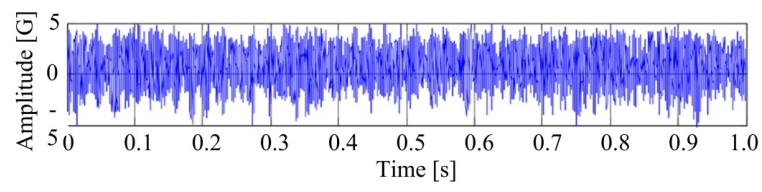

Fig. 10. The strong noise interference in the real working conditions

The SNR can be estimated after extracting strong noise interference in real working conditions, and its calculation process is shown in Eq. (15).

$$
E P R=10 \lg (S E / N E)=10 \lg \left(\sum_{i=1}^{I} s_{i}^{2} / \sum_{i=1}^{I} n_{i}^{2}\right),
$$

where $E P R$ is the SNR in real working condition, $S E$ the energy of useful signal, $N E$ the energy of strong noise interference, and $I$ the number of data points.

The gear fault vibration signal in strong noise interference is obtained by adding strong noise interference after energy conversion into the vibration signal collected from the mechanical fault simulation bench. In this paper, the mechanical fault simulation bench is composed of motor, planetary gearbox, spur gearbox, load system and vibration sensors, and vibration sensors are arranged on the housing of a planetary gearbox. In the experimental process, the broken tooth fault of sun gear in the planetary gearbox is simulated, and the broken tooth fault is processed by wire-cutting technology. The length of the broken tooth is $7 \mathrm{~mm}$, and the broken gear is installed in the planetary gearbox. The motor speed is set to $40 \mathrm{~Hz}$, and the load is set to $13.5 \mathrm{Nm}$ by control software. The acquisition system works in synchronous sampling mode, and the sampling frequency is set to $12800 \mathrm{~Hz}$. The collected experiment with the mechanical fault simulation bench is shown in Fig. 11, and the collected gear fault vibration signal is shown in Fig. 12.

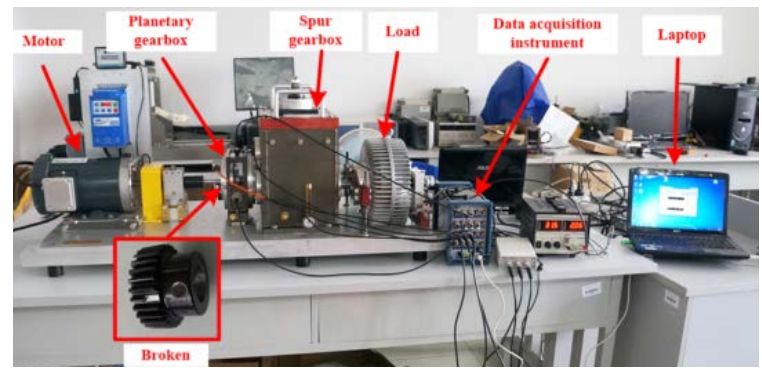

Fig. 11. The collected experiment and gear state

According to the SNR in real working conditions, the energy conversion coefficient can be confirmed. The noise interference in real working conditions converted by energy conversion coefficient is added 
into the gear fault vibration signal collected from the mechanical fault simulation bench. The gear fault vibration signal under strong noise interference is shown in Fig. 13, from which it can be seen that the gear fault vibration signal is all submerged after adding the strong noise interference in real working conditions, and its frequency spectrum is shown in Fig. 14.

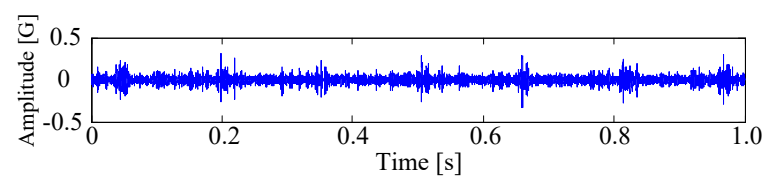

Fig. 12. Vibration signal of broken gear fault collected from mechanical fault simulation bench

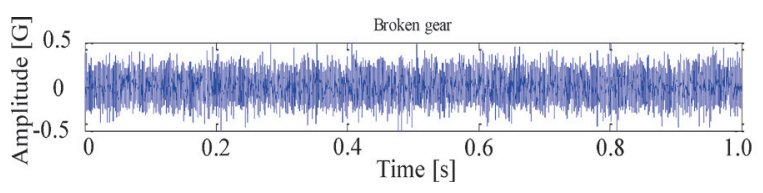

Fig. 13. The gear fault vibration signal under strong noise interference in real working condition

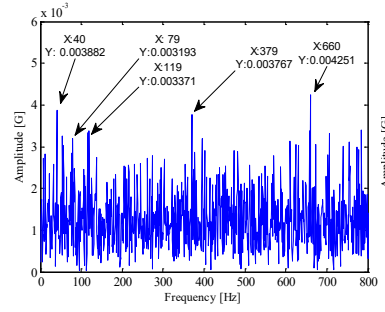

a)

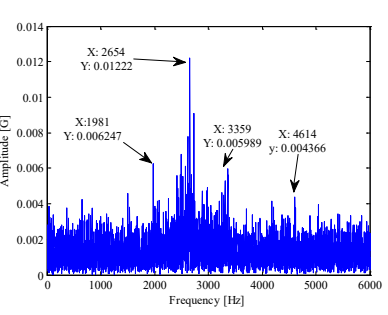

b)
Fig. 14. The frequency spectrum of gear fault vibration signal in strong noise interference; a) detail frequency spectrum, and b) full frequency spectrum

It can be seen from Fig. 14 that the prominent frequency points in the detail frequency spectrum are $40 \mathrm{~Hz}, 79 \mathrm{~Hz}$, and $119 \mathrm{~Hz}$, which are the output frequency of the motor and its double frequency and triple frequency, respectively. However, there are also high amplitude frequency components around these frequencies, which cause interference to these feature frequencies. Furthermore, $660 \mathrm{~Hz}$ corresponds approximately to the meshing frequency of the gear system, which is the installation part of the fault gear, and $1981 \mathrm{~Hz}, 2654 \mathrm{~Hz}, 3359 \mathrm{~Hz}$ and $4614 \mathrm{~Hz}$ correspond to the triple frequency, fourfold frequency, fivefold frequency, and sevenfold frequency, respectively. Although some frequency points are prominent in the frequency spectrum but the gear fault vibration signal suffers from strong noise interference in the low-, middle-, and high-frequency bands. Next, the gear fault vibration signal is decomposed by DTCWT, and the decomposition layer is set to 6. In the decomposition process of DTCWT, the applied filters are as follows: The high-pass filter $\mathbf{h}_{1}$ of real tree is $[-0.00455,0.00543,0.01702,-0.02382$, $-0.10671,-0.01186,0.56881,-0.75614,0.27529$, $0.11720,-0.03887,-0.03466,-0.00388,-0.00325]$, the low-pass filter $\mathbf{h}_{0}$ of real tree is $[0.00325$, $-0.00388,0.03466,-0.03887,-0.1172,0.27529$, $0.75614,0.56881,0.01186,-0.10671,0.02382$, $0.01702,-0.00543,-0.00455]$. The high-pass filter $\mathbf{g}_{1}$ of complex tree is $[-0.00325,-0.00388,-0.03466$, $-0.03887,0.11720,0.27529,-0.75614,0.56881$, $-0.01186,-0.10671,-0.02382,0.01702,0.00543$, $-0.00455]$, and the low-pass filter $\mathbf{g}_{0}$ of complex tree is $[-0.00456,-0.00544,0.01702,0.02382,-0.1067$, $0.01186, \quad 0.56881,0.75614,0.27529,-0.11720$, $-0.03887,0.03466,-0.00388,0.00325]$. The decomposition result of DTCWT is shown in Fig. 15.
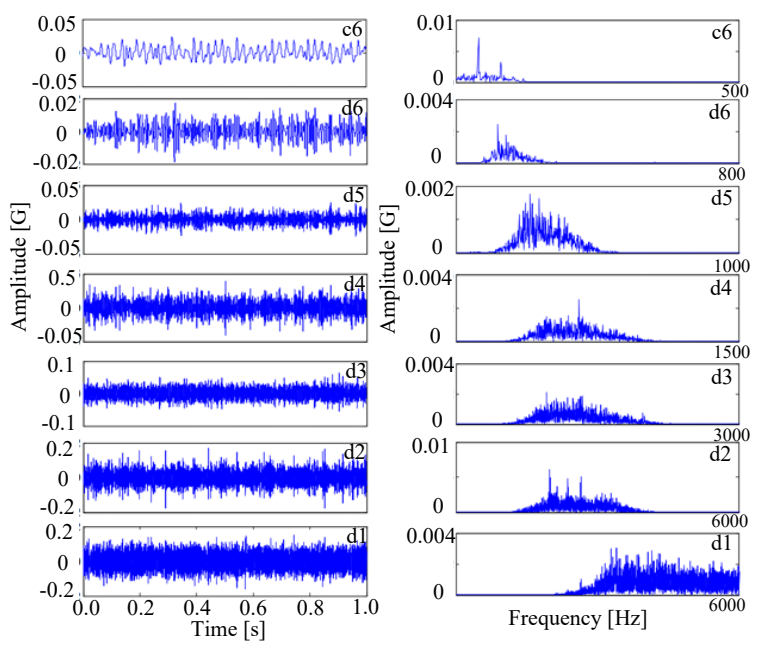

Fig. 15. The decomposition result of DTCWT

With DTCWT, seven signal components with different frequency bands and different resolution attributes are obtained. Meanwhile, the strong noise interference is also decomposed into different band signals according to their attributes. For the obtained signal components with different frequency bands, the noise reduction based on cyclic singular energy difference spectrum is carried out. Because the termination condition of the proposed noise reduction method is that the denoising order determined by the last cyclic is less than a certain value, different cycle termination conditions can be set for different band signals. In this paper, by comparing several experiments, the termination condition of noise reduction of frequency band $\mathrm{c} 6$ to $\mathrm{d} 1$ is that the denoising order determined by the last cycle is less 
than $[30,50,50,80,100,100,120]$, respectively. Due space limitations, the noise reduction process of frequency band $\mathrm{d} 2$ layer is illustrated as an example. The noise reduction process is shown in Fig. 16.

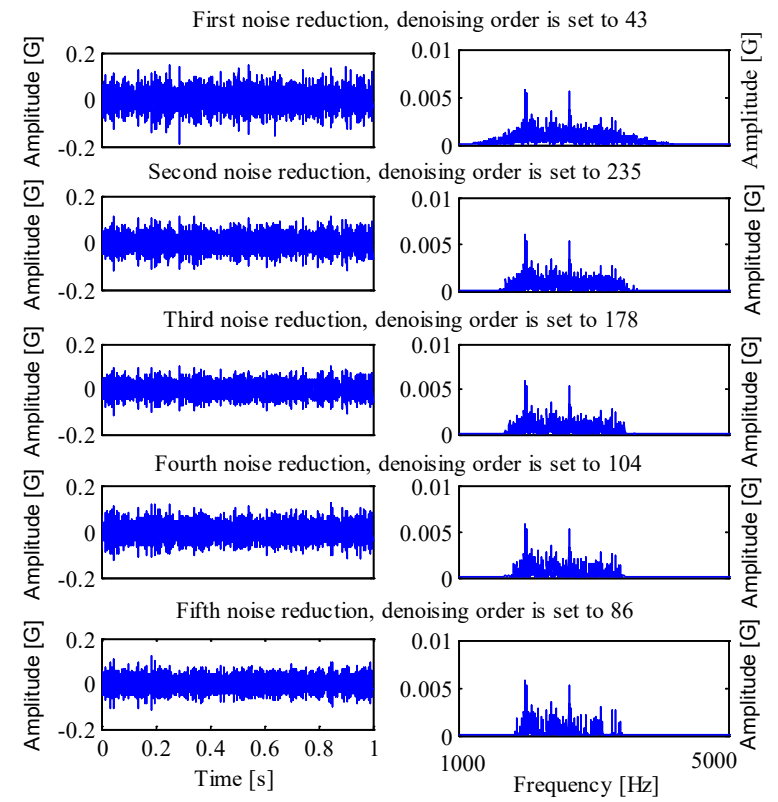

Fig. 16. Noise reduction process of frequency band $d 2$

It can be seen from Fig. 16 that the noise reduction process of frequency band $\mathrm{d} 2$ is completed after 5 cycles of noise reduction. Compared to the signal of frequency band $\mathrm{d} 2$ layer between Figs. 15 and 16, the signal of frequency band $\mathrm{d} 2$ layer in Fig. 15 contains more noise interference, and the noise interference submerges most of the original signal frequency components. Based on the idea of the cascaded cycle and successive elimination of noise interference, the proposed cyclic singular energy difference spectrum is used, and the signal components determined to be noise interference are filtered out step by step in the process of each cycle noise reduction. It can also be seen from Fig.16 that the noise interference can be eliminated step by step with the increasing of the cycle number of noise reduction; then, the strong noise interference in frequency band $\mathrm{d} 2$ layer can be eliminated, and the useful signal can be retained.

To analyse the effect of different delay parameters on the noise reduction result, and the mutual information function method is proved to be effective in selecting the delay parameter, some qualitative and quantitative analyses are carried out. Similarly, the vibration signal of frequency band $\mathrm{d} 2$ is taken as the analysis object, and the delay parameters are set as 2, 4, 6, 8, 10 and 12 , respectively, where the delay parameter is equal to 6 , which is selected by the mutual information function method in this paper. In addition, for different delay parameters, the same methods are used to select the embedding dimension and denoising order. For the vibration signal of frequency band $\mathrm{d} 2$, the noise reduction results using different delay parameters are shown in Fig. 17.

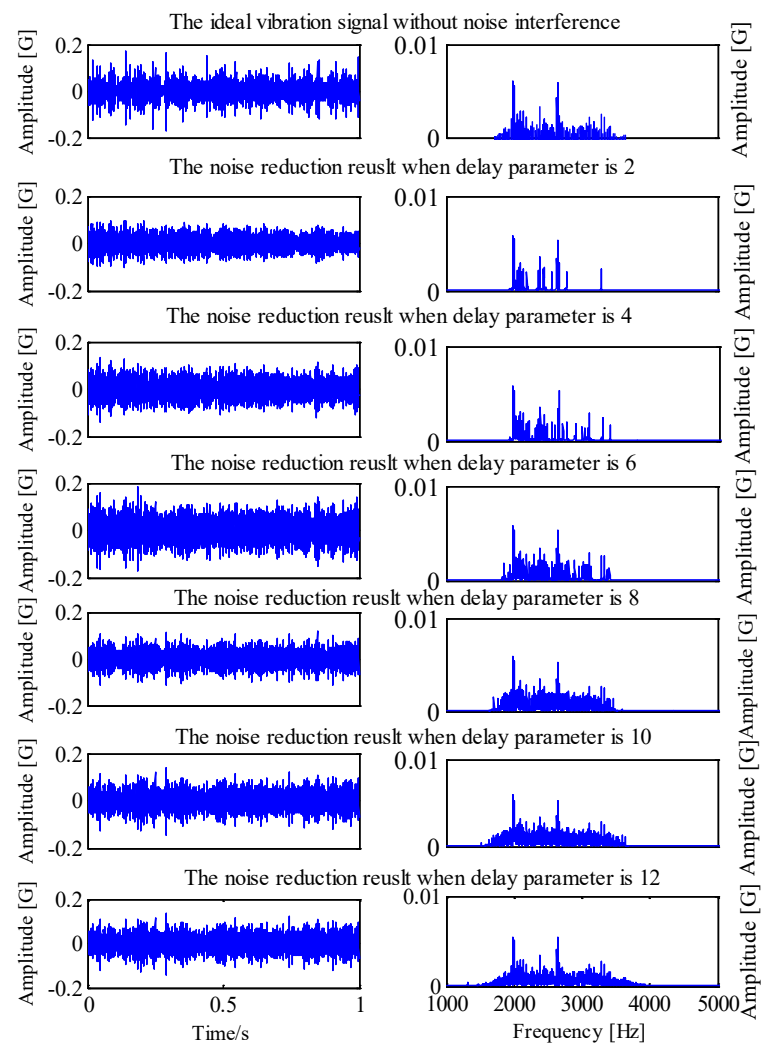

Fig. 17. The noise reduction results using different delay parameters

In the analysis process, except for different delay parameters, the other processing methods are all consistent. As can be seen from Fig. 17, with the increase of the value of the delay parameter, more signal components are retained. Compared with the ideal vibration signal without noise interference, when the delay parameter is selected 2 or 4 , although their noise reduction results retain the main signal components, some useful signals are eliminated as noise interference. When the delay parameter is selected as 10 or 12 , it can be found that there is too much noise interference at both ends of the signal frequency band, and the noise interference can not be effectively eliminated. By comparison, when the delay parameter is selected 6 or 8 , they have a better noise reduction effect. In addition, the noise interference 
Table 1. Some feature indexes of the noise reduction results of different delay parameters and the ideal vibrations signal without noise interference

\begin{tabular}{|c|c|c|c|c|c|c|c|}
\hline Delay parameter & 2 & 4 & 6 & 8 & 10 & 12 & $\begin{array}{c}\text { Vibration signal without } \\
\text { noise interference }\end{array}$ \\
\hline Energy & 3.30 & 4.96 & 9.04 & 11.01 & 13.83 & 14.27 & 9.22 \\
\hline Average value $\left(\times 10^{-6}\right)$ & 1.18 & 9.87 & 4.20 & 9.7 & 4.2 & 3.45 & 6.87 \\
\hline Root mean square & 1.82 & 2.23 & 3.01 & 3.32 & 3.72 & 3.93 & 3.04 \\
\hline Kurtosis & 5.11 & 3.23 & 3.45 & 3.55 & 3.76 & 3.84 & 5.23 \\
\hline Waveform factor & 143.3 & 144.1 & 145.3 & 146.1 & 146.6 & 147.1 & 146.28 \\
\hline Kurtosis factor $\left(\times 10^{-3}\right)$ & 1.4 & 2.7 & 7.2 & 10 & 14.9 & 16.4 & 8.3 \\
\hline
\end{tabular}

results of different delay parameters are quantitatively analysed. Some feature indexes of the noise reduction results of different delay parameters and the ideal vibrations signal without noise interference are calculated, and they are shown in Table 1.

It can be seen from Table 1 that the quantization indexes of noise reduction results are the closest to the vibration signal without noise interference when the delay parameter is 6 or 8 . Because of the better symmetry of the vibration signal, the magnitude of average values is smaller. When the delay parameter is 2 , only the most prominent signal components are retained, and their kurtosis indexes are the largest. When the delay parameter is selected as other values, some abrupt signal components are eliminated by mistake, so the kurtosis index is relatively small, which is also the key issue to be studied in the future. When the delay parameter is set to 12 , it can be found that the energy of the noise reduction signal is higher approximately by $54 \%$ from the vibration signal without noise interference, because when the delay parameter is 12 or another large value, the data in the $(n+1)^{\text {th }}$ row always lags behind more data points compared with the data in the $n^{\text {th }}$ row. This phenomenon causes the dimension of the phase space to decrease, and the size and number of extreme points of the singular energy difference spectrum is also reduced. However, the termination condition in the proposed method is that the maximum sequence number of the singular values whose abrupt extreme values are greater than the average value of those is less than a certain value, so the cycle noise reduction process stops prematurely when the delay parameter is large, and some noise interference is not eliminated. Then, when the delay parameter is 12 , the noise reduction signal still contains some noise interference, so the energy of the noise reduction signal is significantly higher than that of the vibration signal without noise interference. In this paper, the delay parameter selected by the mutual information function method is 6 , which also proves that it is feasible to apply mutual information function method for delay parameter selection.

For other frequency band signals obtained by DTCWT, the noise reduction is carried out according to the above process, and the signals after noise reduction of each frequency band are inversely reconstructed to obtain the final noise reduction result. The final noise reduction results of the gear fault vibration signal and its frequency spectrum are shown in Fig. 18. Fig. 19 is the comparison of the detailed spectrums of the vibration signal without noise interference and the vibration signal with noise interference after noise reduction. Meanwhile, the effectiveness of the proposed method is proved by combining the comparison analysis of the above Fig. 14 , which is the frequency spectrums of the vibration signal in strong noise interference.

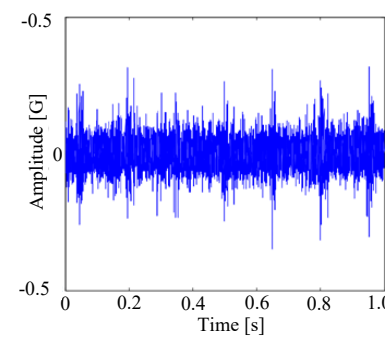

a)

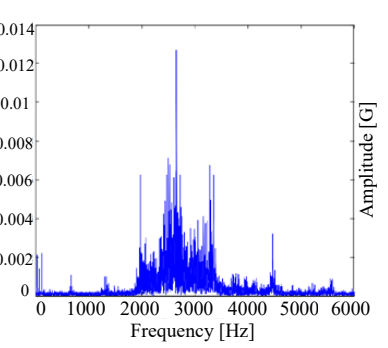

b)
Fig. 18. Final noise reduction result of gear fault vibration signal and its frequency spectrum; a) gear fault vibration signal after noise reduction, and b) frequency spectrum after noise reduction

To compare and analyse the above results comprehensively, it can be found that gear fault vibration signal is disturbed by strong noise interference seriously, main features of gear fault vibration signal are completely submerged by strong noise interference, and the low-, middle-, and highfrequency bands are full of noise interference (Fig. 14). After the processing of the proposed method, the gear fault vibration signal after noise reduction shows the regularity similar to the gear fault vibration signal 
without noise interference, and the noise interference in low-, middle-, and high-frequency bands are greatly reduced (comparison between Figs. 14 and 18). That is because DTCWT is used for signal decomposition, and each signal component obtained by DTCWT is denoised separately. Meanwhile, to compare the detailed spectrums between the vibration signal without noise interference and the vibration signal with noise interference after noise reduction (Fig. 19 ), it can be found that they have the same main frequency components, and the main composition of signal components is also similar. Most noise interference can be eliminated, main signal features can be represented, and the useful signal components can be obtained. However, it also can be found that the proposed noise reduction method does not completely eliminate the strong noise interference in real working condition, and there is still a small amount of noise interference. However, relatively speaking, any noise reduction method can not eliminate all noise interference. Compared with the strong noise interference in real working conditions, the proposed noise reduction method has a relatively effective noise reduction effect.

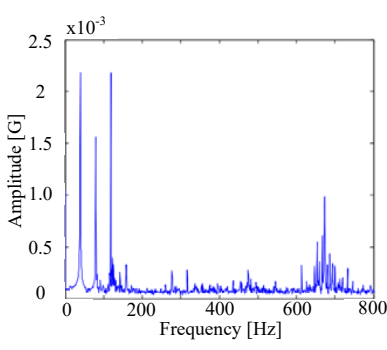

a)

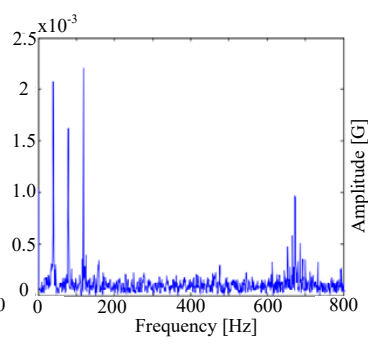

b)
Fig. 19. The comparison of detailed spectrums; a) the detailed spectrum without noise interference, b) the detailed spectrum with noise interference after noise reduction

In order to further illustrate the effectiveness of the proposed method, the proposed method is compared with the existing technologies, and the Butterworth low-pass filter and one-time noise reduction method based on SVD are used to process the same vibration signal under strong noise interference, respectively. When the Butterworth lowpass filter is applied, the cut-off frequencies are set as [80 Hz, $200 \mathrm{~Hz}, 300 \mathrm{~Hz}, 700 \mathrm{~Hz}, 1500 \mathrm{~Hz}, 3000 \mathrm{~Hz}$, $5000 \mathrm{~Hz}]$ for the signal components of frequency band c6-d1 obtained by DTCWT after many experiments, and the final noise reduction result processed by Butterworth low-pass filter is shown in Fig. 20. When the one-time noise reduction method based on SVD is applied, the denoising order orders are set as [30,
$50,50,80,100,100,120]$ for the signal components of frequency band c6-d1 obtained by DTCWT, which is consistent with the proposed method in this paper. The final noise reduction result processed by one-time noise reduction method based on SVD is shown in Fig. 21.

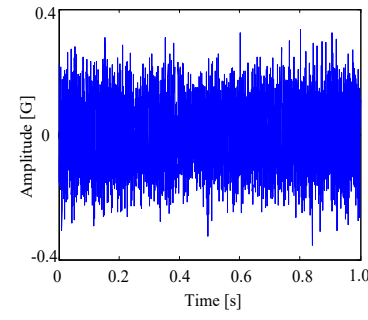

a)

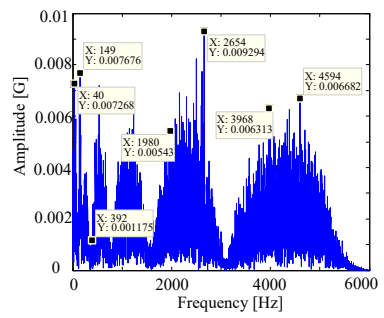

b)
Fig. 20. Final noise reduction result processed by Butterworth low-pass filter; a) time-domain signal after noise reduction, and b) frequency spectrum after noise reduction

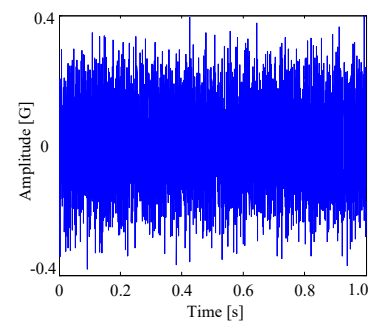

a)

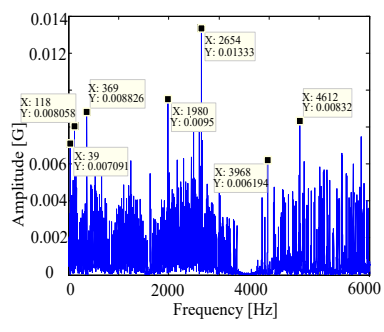

b)
Fig. 21. Final noise reduction result processed by one-time noise reduction method based on SVD; a) time-domain signal after noise reduction, and b) frequency spectrum after noise reduction

It can be seen from Fig. 20 that the time-domain signal processed by the Butterworth low-pass filter has no characteristic to follow. From the frequency spectrum, there is still more noise interference in the low-, middle-, and high-frequency bands. Among the frequency salient points marked in Fig. 20, some of them are the feature information contained in the original signal, but most of them are not included in the original signal. The noise reduction effect processed by Butterworth low-pass filter is worse than that of the proposed method. The main reason is that the low-pass filter eliminates all signal components lower than the cut-off frequency, and retains all signal components higher than the cut-off frequency. The strong noise interference in real working conditions exists in all frequency bands, so it is inevitable that the noise interference in some frequency bands cannot be eliminated.

Furthermore, it is very difficult to set the cut-off frequency reasonably when dealing with the actual noise interference. It can be seen from Fig. 21 that 
the time-domain signal processed by one-time noise reduction method based on SVD does not reflect the regular shock waveform similar to that in Fig. 18. In the frequency spectrum of Fig. 21, the main features of the vibration signal (marked in Fig. 21) are retained, but their clarity is far less than that in Fig. 18. there are still more noise interferences in the low-, middle-, and high-frequency bands of the whole frequency spectrum, which will interfere with these main feature information points.

Moreover, it also can be found that the signal components in the frequency band from $3000 \mathrm{~Hz}$ to $4000 \mathrm{~Hz}$ are eliminated as noise interference, and the main features from $3000 \mathrm{~Hz}$ to $4000 \mathrm{~Hz}$ are also mistaken as noise interference, which is a bad result. It can be seen that the noise reduction result processed by one-time noise reduction method based on SVD is far worse than that processed by the proposed method. The above comparison and analysis can also show that the proposed method has better noise reduction effect.

In this paper, the proposed method can be used to eliminate the strong noise interference of the vibration signal of equipment. It is a pre-processing for gear fault diagnosis process, which ensured that the further feature extraction and fault recognition can be conducted effectively and smoothly. Also, the proposed noise reduction method can also be applied to the detection of gear quality for production process. The authors of the present paper assert that the proposed method can be successfully applied to gear production process through two ways: The first way is that the proposed noise reduction method is applied to the condition monitoring of gear cutting machine. The vibration signal of the gear cutting machine can be obtained, and the proposed method can eliminate the noise interference contained in the vibration signal. The feature information that can reflect the operating state of the gear-cutting machine can be retained, and the operating state of the gear-cutting machine can be evaluated. The cutting quality of gear production process can be further guaranteed.

Another way is that the proposed noise reduction method is applied to the gear quality test after the gear is machined. A special test bench for testing the quality of gears can be built, and the machined gears can be run on the test bench. The vibration signal of the test bench can be obtained, and the proposed noise reduction method can be used to process the vibration signal, the useful signals which can be reflected in the gear quality being obtained by using the proposed method to eliminate the external interference. Further combined with other signal-processing technologies, the gear machining defects, such as excessive radial runout, tooth surface defects, tooth profile asymmetry and tooth profile periodic error, can be detected. Through the above two ways, the noise reduction method proposed in this paper can be applied to the gear production process to ensure the quality of the gear.

\section{CONCLUSIONS}

A pre-treatment noise reduction method based on DTCWT and cyclic singular energy difference spectrum is proposed in this paper. DTCWT can be used to decompose the gear fault vibration signal under strong noise interference, and the noise interference with different frequency attributes can be decomposed into different signal components. Based on the noise reduction principle of SVD, cyclic singular energy difference spectrum is proposed. According to the characteristics of each signal components obtained by DTCWT, the noise interference can be purposefully eliminated successively.

Furthermore, the termination condition of cyclic singular energy difference spectrum can be set according to the noise interference distribution characteristics in different frequency bands, and it has a better noise reduction effect than the one-time noise reduction idea. The final noise reduction of gear fault vibration signal under noise interference can be realized on the basis of reducing noise for all signal components obtained by DTCWT. The experiment results show that the proposed method can effectively eliminate the strong noise interference in real working condition, and the useful vibration signal components can be retained.

\section{ACKNOWLEDGEMENTS}

This research was funded by National Natural Science Foundation of China (51905147), Changzhou Sci \& Tech Program (CJ20190055), the Fundamental Research Funds for the Central Universities (B200202226), and the Special project of science and technology innovation of Tiandi Science \& Technology (2018-TD-MS033), and is gratefully acknowledged.

\section{REFERENCES}

[1] Pang, X.Y., Cheng, B.A., Yang, Z.J. (2019). A fault feature extraction method for a gearbox with a composite gear train based on EEMD and translation-invariant multiwavelet neighbouring coefficients. Strojniski vestnik - Journal of 
Mechanical Engineering, vol. 65, no. 1, p. 3-11, D0l:10.5545/ sv-jme.2018.5441.

[2] Mohammad, R.A.A.A., Ashkan, M., Meghdad, K. (2016). Wavelet transform and least square support vector machine for mechanical fault detection of an alternator using vibration signal. Journal of Low Frequency Noise Vibration and Active Control, vol. 35, no. 1, p. 52-63, DOI:10.1177/0263092316628258.

[3] Klausen, A., Khang, H.V., Robbersmyr, K.G. (2020). Multi-band identification for enhancing bearing fault detection in variable speed conditions. Mechanical Systems and Signal Processing, vol. 139, art. ID:106422, D0l:10.1016/j.ymssp.2019.106422.

[4] Brigham, K., Zappal, D., Crabtree, C.J., Donaghy, S.C. (2020). Simplified automatic fault detection in wind turbine induction generators. Wind Energy, vol. 23, no. 4, p. 1135-1144, DOI:10.1002/we.2478.

[5] Tsiaparas, N.N., Golemati, S., Andreadis, I., Stoitsis, J., Valavanis. I., Nikita, K.S. (2013). Assessment of carotid atherosclerosis from B-mode ultrasound images using directional multi-scale texture features. Measurement Science and Technology, vol. 23, 114004, D0I:10.1088/09570233/23/11/114004.

[6] Mamun, Md., Al-Kadi, M., Marufuzzamann, M. (2013). Effectiveness of wavelet denoising on electroencephalogram signals. Journal of Applied Research and Technology, vol. 11, no. 1, p. 156-160, D0I:10.1016/S1665-6423(13)71524-4.

[7] Zhao, M., Jia, X. (2017). A novel strategy for signal denoising using reweighted SVD and its applications to weak fault feature enhancement of rotating machinery. Mechanical Systems and Signal Processing, vol. 94, p. 129-147, D0l:10.1016/j. ymssp.2017.02.036.

[8] Jiang, H.M., Chen, J., Dong, G.M. (2015). Study on hankel matrix-based SVD and its application in rolling element bearing fault diagnosis. Mechanical Systems and Signal Processing, vol. 52, p. 338-359, D0l:10.1016/j.ymssp.2014.07.019.

[9] Tomassi, D., Milone, D., Nelson, J.D.B. (2015). Wavelet shrinkage using adaptive structured sparsity constraints. Signal Processing, vol. 106, p. 73-87, D0l:10.1016/j. sigpro.2014.07.001.

[10] Dudik, J.M., Coyle, J.L., El-Jaroudi, A., Sun, M., Sejdić, E. (2016). A matched dual-tree wavelet denoising for tri-axial swallowing vibrations. Biomedical Signal Processing and Control, vol. 27, p. 112-121, D0l:10.1016/j.bspc.2016.01.012.

[11] Yu, X., Liang, W., Zhang, L., Jin, H., Qiu, J. (2016). Dual-tree complex wavelet transform and SVD based acoustic noise reduction and its application in leak detection for natural gas pipeline. Mechanical Systems and Signal Processing, vol. 72 73, p. 266-285, D0l:10.1016/j.ymssp.2015.10.034.

[12] Yin, M., Liu, W., Zhao, X. (2013). Image denoising using trivariate prior model in nonsubsampled dual-tree complex contourlet transform domain and non-local means filter in spatial domain. Optik, vol. 124, no. 24, p. 6896-6904, D0l:10.1016/j.ijleo.2013.05.132.

[13] Chen, X.H., Cheng, G., Li, Y. (2018). Fault diagnosis of planetary gear based on entropy feature fusion of DTCWT and OKFDA. Journal of Vibration and Control, vol. 24, no. 21, p. 5044-5061, D0I:10.1177/1077546317742506.

[14] Xu, Y., Meng, Z., Lu, M. (2014). Gear fault diagnosis based on dual-tree complex wavelet transform and singular value difference spectrum. Journal of Vibration and Shock, vol. 33, no. 1, p. 11-16.

[15] Kingsbury, N.G. (1998). The dual-tree complex wavelet transform: a new technology for shift invariance and directional filters. IEEE Digital Signal Processing Workshop, vol. 98 , no. 1, p. 2-5.

[16] Traore, O.L., Pantera, L., Favretto-Cristini, N., Cristinni, P., Viguier-Pla, S., Vieu, P. (2017) Structure analysis and denoising using singular spectrum analysis: Application to acoustic emission signals from nuclear safety experiments. Measurement, vol. 104, p. 78-88, D0l:10.1016/j. measurement. 2017.02.019.

[17] De, M.B. (1993). The singular value decomposition and long and short spaces of noisy matrices. IEEE Transactions on Signal Processing, vol. 41, no. 9, p. 2826-2838, DOI:10.1109/78.236505.

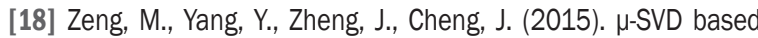
denoising method and its application to gear fault diagnosis. Chinese Journal of Mechanical Engineering, vol. 51, no. 3, p. 95-103, D0l:10.3901/JME.2015.03.095. (in Chinese)

[19] Wang, B., Ren, Z., Wen, B. (2012). Fault diagnosis method of rotating machines based on nonlinear multi-parameters. Chinese Journal of Mechanical Engineering, vol. 48, no. 5, p. 63-69, D0I:10.3901/JME.2012.05.063.

[20] Cao, L.Y. (1997). Practical method for determining the minimum embedding dimension of a scalar time series. Physica D: Nonlinear Phenomena, vol. 110, no. 1-2, p. 43-50, DOl:10.1016/s0167-2789(97)00118-8.

[21] Cong, F.Y., Chen, J., Dong, G.M., Zhao, F. (2013). Short-time matrix series based singular value decomposition for rolling bearing fault diagnosis. Mechanical Systems and Signal Processing, vol. 34, no. 1-2, p. 218-230, D0l:10.1016/j. ymssp.2012.06.005. 